\title{
Effect of elastic domains on electromechanical response of epitaxial ferroelectric films with a three-domain architecture
}

\author{
Jun OUYANG ${ }^{a,}$, Wei ZHANG ${ }^{a}$, S. Pamir ALPAY ${ }^{b}$, Alexander L. ROYTBURD ${ }^{c, *}$ \\ ${ }^{a}$ Key Laboratory for Liquid-Solid Structural Evolution and Processing of Materials (Ministry of Education), \\ Engineering Ceramics Laboratory, School of Materials Science and Engineering, \\ Shandong University, Jinan 250061, Shandong, China \\ ${ }^{b}$ Materials Science and Engineering Program and Institute of Materials Science, University of Connecticut, \\ Storrs 06269, Connecticut, USA \\ ${ }^{c}$ Department of Materials Science and Engineering, University of Maryland, College Park 20742, Maryland, USA
}

Received: January 28, 2013; Accepted: January 31, 2013

(CThe Author(s) 2013. This article is published with open access at Springerlink.com

\begin{abstract}
Thermodynamics of (001) epitaxial ferroelectric films completely relaxed due to the formation of elastic domains with a three-domain architecture is presented. The polydomain structure and electromechanical response of such films are analyzed for two cases corresponding to immobile and mobile elastic domain walls. It is shown that immobile elastic domains provide additional constraint which increases the mechanical and electrical clamping, thereby significantly reducing the piezoelectric and dielectric responses. On the other hand, a polydomain ferroelectric film adapts to the variations in the applied electric field by reversible domain wall displacements in the case of mobile domain walls. The comparison of the theory with experiments shows that the elastic domain walls are mobile in the fully relaxed films of $\sim 1 \mu \mathrm{m}$ thickness. In addition, if the substrate constraint is reduced via decreasing lateral size of a polydomain ferroelectric film, its piezoresponse will increase dramatically, as is experimentally verified on small islands of polydomain ferroelectric films. The general conclusions can be readily applied to other constrained polydomain films.
\end{abstract}

Keywords: ferroelectrics; domains; epitaxial films; domain wall; thermodynamics; electromechanical properties

\section{Introduction}

The formation of elastic domains is a universal mechanism of relaxing the internal stresses in constrained phase transformation in solids [1-3]. The arrangements of elastic domains or the polydomain structure determine most of the physical properties of

* Corresponding authors.

E-mail: J. Ouyang, ouyangjun@sdu.edu.cn

A. L. Roytburd, roytburd@umd.edu the product phase. Particularly, the switching of elastic domains increases the compliance of polydomain phases to external mechanical, electrical or magnetic fields. The concept of elastic domains has been successfully applied to the analysis of polydomain microstructures in epitaxial films [4], and numerous polydomain structures in epitaxial films of superconductors [5], semiconductors [6], ferromagnetic [7] and ferroelectrics [8-15] have been studied experimentally and theoretically.

In recent years, ferroelectric thin films have become an object of intensive research because of their 
potential applications not only as non-volatile random access memories or dynamic random access memories, but also as active components of sensors, actuators and micro-electromechanical systems (MEMS). These applications require either a high electric field response to an external mechanical field (direct piezoeffect) for sensors, or a high strain response to an applied electric field (converse piezoeffect) for actuators and MEMS. However, the fundamental problem that ferroelectric films have inferior electrical and electromechanical properties compared to their bulk counterparts limits their use. One particular example is the substantial reduction of the piezoelectric responses due to the substrate clamping of single domain epitaxial ferroelectric films [16]. To overcome the limit set by the substrate clamping on the intrinsic electromechanical properties, it is necessary to utilize the extrinsic effects from the movement of boundaries between elastic (non- $180^{\circ}$ ) domains.

Since the formation of elastic domains is a mechanism of relaxing a film/substrate heterostructure to approach equilibrium, it is possible to shift the equilibrium by applying an electric field and obtain reversible field-induced deformation due to the change of domain structure [17]. The elastic domain structure in epitaxial films has been studied extensively in theory [11,18-22] and in experiment [12,13,23] during the last two decades. However, the theoretical work, including the comprehensive analysis based on homogeneous theory $[10,18]$, construction of domain stability map based on theories of elastic domains and coherent phases [19], phase field modeling [11], and exact analytical solutions [20,21], mainly concentrated on elastic domain assemblies consisting of two domain variants (polytwins), except a few by Slutsker et al. [22].

For a (001)-oriented film, which is of most technological significance due to its compatibility with industrial processes, e.g., CMOS process on a (100) $\mathrm{Si}$ substrate, the two-domain structures are able to relax misfit stress only partially, reducing biaxial stress to a uniaxial one. The complete relaxation, in general, requires the formation of polydomain structures including more than two types of possible domains. For example, in the case of a cubic-tetragonal transformation, the complete relaxation is obtained if all the three possible domains of the tetragonal phase are involved in the formation of a polydomain structure. The three-domain structures are thermodynamically more preferable than two-domain ones provided films are not very thin $[4,24,25]$. The three-domain structures have been observed in ferroelectric tetragonal $\mathrm{PbZrTiO}_{3}[26]$ and $\mathrm{BaTiO}_{3}[14]$ films with thickness of more than $300 \mathrm{~nm}$, and it has been shown that the misfit stress in these films is close to zero. Since the equilibrium three-domain films are stress-free in the absence of an external electric field and the field-induced deviation of structure and polarization from the equilibrium are small, the linear theory is adequate for analyzing their electromechanical properties. It allows us to obtain theoretical conclusions in a simple form, which can be easily applied to the interpretation of the experimental results.

In this paper, a thermodynamic analysis of constrained ferroelectric films is presented to explain the piezoeffect in (001) films with three-domain architecture of elastic domains. Results of theoretical calculations are compared with those from experimental studies on epitaxial (001) $\mathrm{PbZr}_{0.2} \mathrm{Ti}_{0.8} \mathrm{O}_{3}$ (PZT 20/80) films to verify the fundamental concepts. It is clearly demonstrated that elastic domains can be sufficiently mobile in constrained ferroelectric films, and the relatively small extrinsic piezoeffect in the epitaxial films can be explained by elastic interactions between the clamped film and substrate. The extrinsic piezoeffect can be increased significantly by reducing the elastic clamping, as is supported by the experimental results on polydomain ferroelectric thin film "islands" with a lateral size comparable to their thickness [13,23].

\section{Thermodynamics of three-domain film in the absence of electric field}

We consider the transformation from a cubic paraelectric phase to a tetragonal ferroelectric phase, which is quite generic and describes the transformation in the PZT 20/80 films. The transformation is accompanied by a self-strain that can be characterized by the following tensors for $a_{1^{-}}, a_{2^{-}}$and $c$-domains, respectively (Fig. 1(a)):

$$
\bar{\varepsilon}_{1}^{0}=\left(\begin{array}{ccc}
\varepsilon_{c} & 0 & 0 \\
0 & \varepsilon_{a} & 0 \\
0 & 0 & \varepsilon_{a}
\end{array}\right), \bar{\varepsilon}_{2}^{0}=\left(\begin{array}{ccc}
\varepsilon_{a} & 0 & 0 \\
0 & \varepsilon_{c} & 0 \\
0 & 0 & \varepsilon_{a}
\end{array}\right), \quad \bar{\varepsilon}_{3}^{0}=\left(\begin{array}{ccc}
\varepsilon_{a} & 0 & 0 \\
0 & \varepsilon_{a} & 0 \\
0 & 0 & \varepsilon_{c}
\end{array}\right)
$$

where $\quad \varepsilon_{a}=\frac{a-a_{0}}{a}=Q_{12} P^{2} ; \quad \varepsilon_{c}=\frac{c-a_{0}}{a}=Q_{11} P^{2} ; \quad Q_{12}=$ 
$d_{31} / 2 P_{0} \chi$ and $Q_{11}=d_{33} / 2 P_{0} \chi$. Here $a$ and $c$ are the lattice parameters of the unconstrained film in the ferroelectric phase at a temperature $T$ below the transformation temperature $T_{\mathrm{c}} ; a_{0}$ is the lattice parameter of the film in the cubic paraelectric phase extrapolated to $T ; Q_{12}$ and $Q_{11}$ are the electrostrictive coefficients in Voigt notation; $P$ is the polarization; $P_{0}$ is the spontaneous polarization in the absence of electric field; $\chi$ is the dielectric susceptibility and $d_{31}$ and $d_{33}$ are the bulk piezomoduli of single domain ferroelectrics. For a ferroelectric film grown epitaxially on a thick cubic substrate such as $(001)_{\text {film }} / /(001)_{\text {substrate }}$, biaxial misfit is symmetrical (Fig. 1(b)); then the fractions of $a_{1^{-}}$and $a_{2}$-domains are equal, and the average self-strain is $\bar{\varepsilon}(\alpha)=(1-\alpha) \bar{\varepsilon}_{3}^{0}+\frac{\alpha}{2}\left(\bar{\varepsilon}_{1}^{0}+\bar{\varepsilon}_{2}^{0}\right)$, where $\alpha$ is the fraction of $a$-domains. The components of $\bar{\varepsilon}(\alpha)$ are $\varepsilon_{1}(\alpha)=\varepsilon_{2}(\alpha)=\varepsilon_{a}+\frac{1}{2} \alpha\left(\varepsilon_{c}-\varepsilon_{a}\right), \quad \varepsilon_{3}(\alpha)=$ $\varepsilon_{c}-\alpha\left(\varepsilon_{c}-\varepsilon_{a}\right)$. The free energy of the polydomain film is

$$
F(\alpha, P)=F_{0}(P)+\left[\varepsilon_{\mathrm{M}}^{0}+\varepsilon_{1}(\alpha)\right]^{2} / S(\alpha)
$$

where $F_{0}(P)$ is the macroscopic free energy of unstressed ferroelectrics, which is usually described by Landau-Devonshire expansion; $S(\alpha)$ is the average elastic compliance which can be approximately determined as the sum of compliances of $c$-domains and $a_{1} / a_{2}$ domains:

$$
S(\alpha)=(1-\alpha)\left(S_{11}+S_{12}\right)+\alpha\left[\left(S_{11}+S_{33}\right) / 2+S_{13}\right]
$$

where $S_{i j}$ are the elastic compliances of the tetragonal phase at constant polarization. Since the elastic

(a)

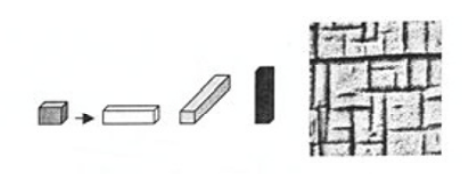

(b)

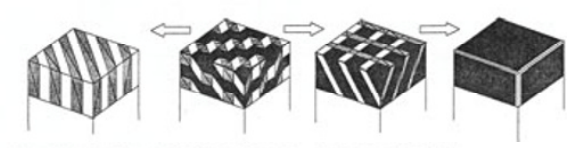

(c)

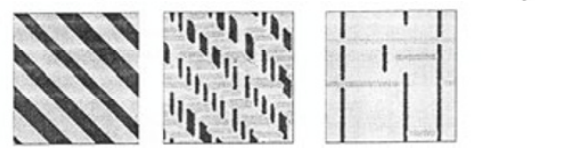

Fig. 1 (a) From left to right, the cubic paraelectric phase, the three ferroelectric tetragonal variants, and the atomic force microscopy image of the cellular domain structure existing in PZT thick films; (b) schematic evolution of the domain architecture as a function of the $a$-domain fraction; (c) phase-field modeling of domain evolution. anisotropy at constant polarization is much less than elastic anisotropy at constant field, the rule of mixtures used in Eq. (3) is approximately correct for small stress. The elastic energy equals to zero for

$$
\alpha_{0}=-\frac{2 \varepsilon_{\mathrm{M}}^{0}}{\varepsilon_{c}-\varepsilon_{a}}-\frac{2 \varepsilon_{a}}{\varepsilon_{c}-\varepsilon_{a}}=-\frac{2 \varepsilon_{\mathrm{M}}^{0}}{\left(Q_{11}-Q_{12}\right) P^{2}}+\frac{2 \delta}{1+\delta}
$$

where $\delta=-Q_{12} / Q_{11}=-d_{31} / d_{33} ; \quad \varepsilon_{\mathrm{M}}^{0}=\left(a_{\mathrm{s}}-a_{0}\right) / a_{0}$ is the misfit between the substrate and film in the cubic paraelectric state extrapolated to the temperature of the ferroelectric film. $a_{\mathrm{s}}$ is the effective lattice parameter that takes into account the relaxation by misfit dislocations $[17,27]$. The relation between the misfit $\varepsilon_{\mathrm{M}}^{0}$ of the paraelectric cubic phase and the misfit of $c$-domains of the ferroelectric tetragonal phase is $c_{\mathrm{M}}=\left(a_{\mathrm{s}}-a\right) / a=\varepsilon_{\mathrm{M}}^{0}+\varepsilon_{a}$. It is worthwhile to note that if the film is completely relaxed by misfit dislocations in its paraelectric state and the difference between thermal expansion coefficients of the film and the substrate is small as well, the film-substrate couple is stress-free just before the structural phase transformation. In this case, the $a$-domain fraction enabling complete relaxation of the misfit stress is reduced to

$$
\alpha_{0}^{*}=-2 d_{31} /\left(d_{33}-d_{31}\right)=2 \delta /(1+\delta)
$$

This domain fraction does not depend on domain polarization and consequently does not depend on the electric field or temperature. Therefore, this structure remains to be stress-free after it is cooled down to room temperature. The variation of $\delta$ and the $c$-domain fraction $(1-\alpha)$ as a function of composition in tetragonal $\mathrm{PbZr}_{x} \mathrm{Ti}_{1-x} \mathrm{O}_{3}$ (PZT) solid solutions are shown in Fig. 2, where the theoretically calculated

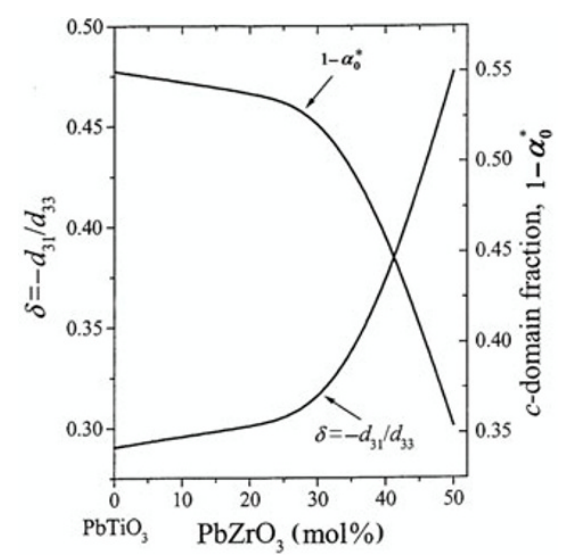

Fig. 2 Variations in $\delta$ and equilibrium $c$-domain fraction $\left(1-\alpha_{0}^{*}\right)$ as functions of zirconium content in tetragonal $\mathrm{PbZr}_{x} \mathrm{Ti}_{1-x} \mathrm{O}_{3}$ solid solutions. 
piezomoduli $d_{31}$ and $d_{33}$ are used [28]. The $c$-domain fraction $(1-\alpha)$ drops from 0.55 to 0.35 as the morph-tropic phase boundary at around $50 \% \mathrm{PbZrO}_{3}$ is approached.

In a more general case, the degree of dislocation relaxation depends on the thickness of the film and $\varepsilon_{\mathrm{M}}^{0} \neq 0$. Then, the stress-free domain structure corresponds to

$$
\alpha_{0}=-2 \varepsilon_{\mathrm{M}}^{0} /\left[\left(Q_{11}-Q_{12}\right) P_{0}^{2}\right]+2 \delta /(1+\delta)
$$

The polarization $P_{0}$ is the equilibrium polarization of the ferroelectric without an external field which corresponds to the minimum free energy $F_{0}(P)$ in Eq. (2) (hereafter, $F_{0}(P)$ is considered as the zero reference state).

As a conclusion, the internal stresses due to lattice misfit can be eliminated in relatively thick films, if all of the three variants of the tetragonal phase are arranged such that the film has the same in-plane size as the substrate. This is based on homogeneous approximation of the film free energy which does not take into account domain architecture [10,29,30].

The architecture of the three-domain structures is dictated by the energy of the interdomain interfaces, the junctions where the three domains come together, and the microstresses at the film/substrate interface. Transmission electron microscopy (TEM) [25] and piezoelectric force microscopy (PFM) $[13,23]$ studies of tetragonal PZT 20/80 films thicker than $300 \mathrm{~nm}$ show that they have a cellular architecture that completely relaxes the internal stresses. An optical microscopy study of epitaxial tetragonal $\mathrm{BaTiO}_{3}$ thick films also illustrates similar morphology of a three-domain structure [14]. These polydomain films are divided into cells of $c$-domains along the (001) direction bound by $a_{1^{-}}$and $a_{2}$-domains of equal domain fraction along the (100) and (010) directions, respectively (Fig. 1(a)). As shown by phase-field modeling, the cellular polydomain structure is favored for small $a$-domain fractions, whereas for relatively larger $a$-domain fractions the second-order arrangement is more stable (Figs. 1(b) and 1(c)) [25]. The modeling also shows that the domain fraction is determined mainly by the trend to minimize misfit stresses, and if the period of domain structure does not exceed the film thickness, the domain fraction can be estimated by using the homogeneous approximation with accuracy better than $\pm 10 \%$.

\section{Effect of applied electric field}

Since the self-strains of ferroelectric phases are functions of the polarization square, the equilibrium domain fraction in Eqs. (4) and (5) does not depend on the presence of $180^{\circ}$ domains. If there are no top and bottom electrodes to compensate free charges, the polydomain structure contains, in addition to the structural $90^{\circ}$ domains, $180^{\circ}$ domains to minimize depolarizing fields. If an electric field $E_{/ /(001)}$ is applied to the polydomain film, the $180^{\circ}$ domains disappear at relatively small field. Under an electric field, two cases are possible for the $90^{\circ}$ domains: (1) $90^{\circ}$ domain walls are immobile, and the polarization and deformation of the film are results of the increase in the saturation polarization inside domains; (2) $90^{\circ}$ domain walls are mobile and contribute to the electric and electromechanical responses (Fig. 3). We consider these two situations and analyze the effects of the polydomain structure on the saturation polarization, dielectric susceptibility, and piezoelectric modulus.

The general expression for the free energy of the constrained film in either case is

$$
\begin{aligned}
F^{\mathrm{f}}= & \frac{\left(P-P_{0}\right)^{2}}{2 \chi(\alpha)}-(1-\alpha) E P \\
& +\frac{1}{S(\alpha)}\left\{\varepsilon_{\mathrm{M}}^{0}+Q_{11} P^{2}\left[-\delta+\frac{1}{2} \alpha(1+\delta)\right]\right\}^{2}
\end{aligned}
$$

where $\chi(\alpha)=\left(\frac{\alpha}{\chi_{11}}+\frac{1-\alpha}{\chi_{33}}\right)^{-1}$ is the effective dielectric

(a)

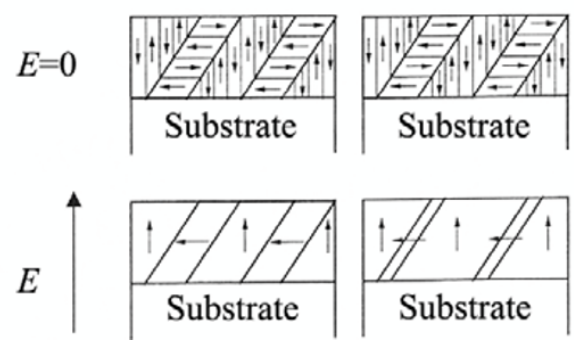

Fig. 3 Two different cases showing schematic domain arrangements due to the applied electric field $E$ : (a) immobile $90^{\circ}$ domain walls and (b) mobile $90^{\circ}$ domain walls, with $180^{\circ}$ domain walls within the polydomains adjust to $E$. The arrows in the domains represent the polarization vectors. 
susceptibility of a free-standing polydomain structure; the second term is the electrostatic energy; the last term is the elastic energy. If the field-induced change of polarization $p=P-P_{0}$, and the change of the domain fraction $\Delta \alpha$, are small as compared with the equilibrium polarization $P_{0}$ and domain fraction $\alpha_{0}$, the free energy can be presented as follows:

$$
\begin{aligned}
& F^{\mathrm{f}}(p, \Delta \alpha)=\frac{1}{2 \chi\left(\alpha_{0}\right)} p^{2}-\left[1-\left(\alpha_{0}+\Delta \alpha\right)\right] E\left(P_{0}+p\right) \\
& \quad+P_{0}^{2} Q_{11}^{2}\left\{2 p\left[\frac{1}{2} \alpha_{0}(1+\delta)-\delta\right]+\frac{1}{2} P_{0} \Delta \alpha(1+\delta)\right\}^{2} / S\left(\alpha_{0}\right)
\end{aligned}
$$

It should be noted that in the absence of the electric field, $F^{\mathrm{f}}=0$ since the elastic energy of the three-domain structure at $\alpha=\alpha_{0}$ is zero.

\section{1 Immobile domain walls}

If the $90^{\circ}$ domain walls are immobile, internal stresses may arise within a polydomain film with the application of an electric field $E_{/ /(001)}$, although in the absence of an electric field the film is stress-free. For $\Delta \alpha=0$, Eq. (6) is reduced to

$$
\begin{aligned}
& F^{\mathrm{f}}(p, \Delta \alpha=0) \\
& \quad=\frac{1}{2 \chi\left(\alpha_{0}\right)} p^{2}-\left(1-\alpha_{0}\right) E\left(P_{0}+p\right)+\frac{1}{S\left(\alpha_{0}\right)}\left[\frac{p}{\chi_{33}} d\left(\alpha_{0}\right)\right]^{2}
\end{aligned}
$$

where $d\left(\alpha_{0}\right)=d_{31}+\frac{1}{2} \alpha_{0}\left(d_{33}-d_{31}\right)$. The minimization of Eq. (7) yields the field-induced change in the polarization:

$$
p(\Delta \alpha=0)=\chi^{\mathrm{f}}(\Delta \alpha=0) E
$$

where, $\chi^{\mathrm{f}}(\Delta \alpha=0)$ is the dielectric susceptibility of the constrained film given by

$$
\chi^{\mathrm{f}}(\Delta \alpha=0)=\left(1-\alpha_{0}\right) \chi_{33}\left\{\frac{\chi_{33}}{\chi\left(\alpha_{0}\right)}+\frac{2\left[d\left(\alpha_{0}\right)\right]^{2}}{\chi_{33} S\left(\alpha_{0}\right)}\right\}^{-1}
$$

For a free standing polydomain film, the field-induced strains correspond to an additional polarization $p$ and are equal to

$$
\begin{gathered}
\Delta \varepsilon_{3}=\varepsilon_{3}(P)-\varepsilon_{3}\left(P_{0}\right)=2 P_{0} Q_{11} p[1-\alpha(1+\delta)] \\
\Delta \varepsilon_{1}=\varepsilon_{1}(P)-\varepsilon_{1}\left(P_{0}\right)=2 P_{0} Q_{11} p\left[-\delta+\frac{1}{2} \alpha(1+\delta)\right]
\end{gathered}
$$

The field-induced strains in constrained films are results of elastic interactions with a substrate, given by $\Delta \varepsilon_{1}^{\mathrm{f}}=\Delta \varepsilon_{1}+\Delta \varepsilon_{1}^{\mathrm{el}}=0, \quad \Delta \varepsilon_{3}^{\mathrm{f}}=\Delta \varepsilon_{3}+\Delta \varepsilon_{3}^{\mathrm{el}}=\Delta \varepsilon_{3}+\xi \Delta \varepsilon_{1}$.
$\xi=-\tilde{S}\left(\alpha_{0}\right) / S\left(\alpha_{0}\right), \quad$ and $\quad \tilde{S}\left(\alpha_{0}\right)=\left[\left(1-\alpha_{0}\right) S_{13}+\alpha_{0} S_{12}\right]$ describes an effective Poisson contraction of a clamping film $[30,31]$. Then, the piezomodulus of the constrained film with immobile domain walls, i.e., $d_{33}^{\mathrm{f}}(\Delta \alpha=0)$, is given by

$$
\begin{aligned}
d_{33}^{\mathrm{f}}(\Delta \alpha=0)=\frac{\mathrm{d} \Delta \varepsilon_{3}^{\mathrm{f}}}{\mathrm{d} E}=\frac{\mathrm{d} \Delta \varepsilon_{3}^{\mathrm{f}}}{\mathrm{d} p} \frac{\mathrm{d} p}{\mathrm{~d} E} \\
=d_{33}\left\{1-\alpha_{0}(1+\delta)+\xi\left[-\delta+\frac{1}{2} \alpha_{0}(1+\delta)\right]\right\} \\
\times\left(1-\alpha_{0}\right)\left\{\frac{\chi_{33}}{\chi\left(\alpha_{0}\right)}+\frac{2\left[d\left(\alpha_{0}\right)\right]^{2}}{\chi_{33} S\left(\alpha_{0}\right)}\right\}^{-1} \\
\approx d_{33}\left\{1-\alpha_{0}(1+\delta)+\xi\left[-\delta+\frac{1}{2} \alpha_{0}(1+\delta)\right]\right\}\left(1-\alpha_{0}\right) \chi\left(\alpha_{0}\right) / \chi_{33}
\end{aligned}
$$

Here the approximation is valid when domain fraction is not very close to 0 or 1 to give $2\left[d\left(\alpha_{0}\right)\right]^{2} /$ $\left[\chi_{33} S\left(\alpha_{0}\right)\right] \ll 1$. The normalized piezomoduli, $d_{33}^{\mathrm{f}}(\Delta \alpha=0) / d_{33}$ for PZT $20 / 80$ polydomain films $\left(\delta=-d_{31} / d_{33}=0.3\right.$ ) are plotted in Fig. 4, by using the approximated expression in Eq. (10), as a function of the $a$-domain fraction $\alpha_{0}$ at different values of $\xi$. This is to clearly demonstrate the central part of the $d_{33}^{\mathrm{f}}\left(\alpha_{0}\right)$ curve $\left(\alpha_{0} \sim \alpha_{0}^{*}\right)$, which is the focus of this investigation. All the curves of $d_{33}^{\mathrm{f}}(\Delta \alpha=0) / d_{33}$ vs. $\alpha_{0}$ intersect at a single point, which corresponds to the domain fraction $\alpha_{0}^{*}=2 \delta /(1+\delta)$, and here $\alpha_{0}^{*}=0.46$ for PZT $20 / 80$. $\alpha_{0}^{*}$ corresponds to the stress-free state at $E=0$ which remains to be stress-free under an electric field, as given by Eq. (4). $d_{33}^{\mathrm{f}}\left(\alpha=\alpha_{0}^{*}, \Delta \alpha=0\right) / d_{33}=0.26$. All other states are stress-free when $E=0$, but become stressed under a field.

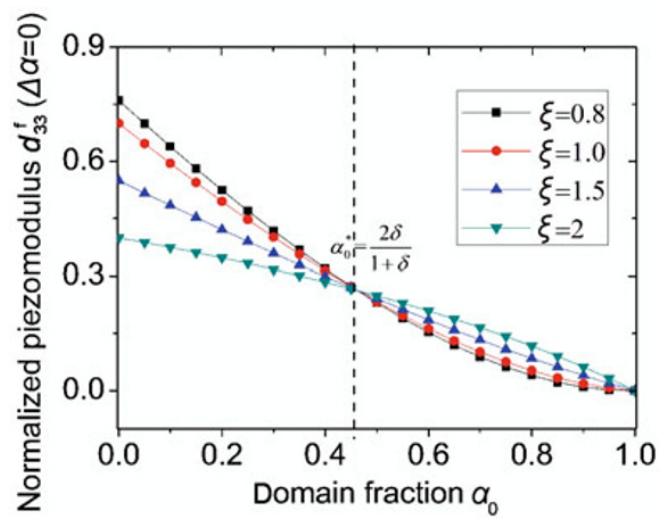

Fig. 4 Normalized piezomodulus of the constrained film $d_{33}^{\mathrm{f}}(\Delta \alpha=0) / d_{33}$ as a function of the $a$-domain fraction $\alpha_{0}$ and $\xi$. 
It should be noted that, by using the approximated expression in Eq. (10), the normalized piezomodulus at a given $\xi$ is only dependent on the ratios of piezomodulus $\delta\left(=-d_{31} / d_{33}\right)$ and dielectric modulus $\kappa\left(=\chi_{33} / \chi_{11}, \quad \chi\left(\alpha_{0}\right) / \chi_{33}=\left[\kappa \alpha_{0}+\left(1-\alpha_{0}\right)\right]^{-1}\right) . \quad$ The domain fraction dependence of $\xi$ is small when $\alpha_{0}$ is near $\alpha_{0}^{*}$, hence it is not taken into account and $\xi$ is chosen as an independent variable. By doing so, most of the material specific parameters in Eq. (10), including $\tilde{S}\left(\alpha_{0}\right), d\left(\alpha_{0}\right)$ and $S\left(\alpha_{0}\right)$, are not built in Fig. 4. Therefore, the major characteristics of the normalized longitudinal piezomodulus are demonstrated in the simple diagram of Fig. 4, for the general case of a constrained polydomain ferroelectric film with immobile domain walls. The realistic values of $d_{33}^{\mathrm{f}}(\Delta \alpha=0)$ are bounded by the curves with $\xi=0.8$ and $\xi=2$ in Fig. 4.

For $\alpha_{0}=\alpha_{0}^{*}, d\left(\alpha_{0}^{*}\right)=0$, the normalized effective piezomodulus of the film does not depend on $\xi$ : $d_{33}^{\mathrm{f}}\left(\alpha_{0}=\alpha_{0}^{*}, \Delta \alpha=0\right) / d_{33}=(1-2 \delta)(1-\delta) \chi\left(\alpha_{0}^{*}\right) /\left[(1+\delta) \chi_{33}\right]$. The normalized piezomodulus of a single $c$-domain film $-d_{33}^{\mathrm{f}}\left(\alpha_{0}=0\right)$ can be simplified to the known relations [30]:

$$
\frac{d_{33}^{\mathrm{f}}\left(\alpha_{0}=0\right)}{d_{33}}=(1-\xi \delta)\left[1+\frac{2 d_{33}^{2} \delta^{2}}{\chi_{33}\left(S_{11}+S_{12}\right)}\right]^{-1}=1-\xi_{E} \delta
$$

where $\xi_{E}=-2 S_{13}^{E} /\left(S_{11}^{E}+S_{12}^{E}\right) ; S_{i j}^{E}$ are the components of elastic compliance at constant field. The drop of the piezomoduli of single-domain ferroelectric films as compared with their bulk counterparts is due to substrate clamping, which has been intensively investigated in literature [15,31-32]. For a polydomain ferroelectric film, in addition to the substrate clamping effect, immobile domain walls provide an additional constraint which further decreases the overall piezoresponse of the film.

\section{2 Mobile domain walls}

If the $90^{\circ}$ domain walls are mobile, the ferroelectric film adapts to the variations in the applied electric field by reversible domain wall displacements. These movements induced by the electric field may contribute significantly to the electrical and electromechanical properties of ferroelectric films [18]. In this case, the application of an electric field $E_{/ /(001)}$ to the polydomain film results in a domain wall motion to decrease the $a$-domain fraction, as well as an increase of polarization inside the domains. The equilibrium polarization and domain fraction follow from the minimization of the free energy given by Eq. (6), i.e., they are solutions of the equations $\partial F / \partial p=0$ and $\partial F / \partial \Delta \alpha=0$. Then, the zero field susceptibility of the constrained polydomain film is

$$
\chi^{\mathrm{f}}(\Delta \alpha \neq 0)=\frac{\mathrm{d} p(\alpha)}{\mathrm{d} E}=\left(\frac{\partial p}{\partial E}\right)_{\alpha=\alpha_{0}}+\left(\frac{\partial p}{\partial \alpha} \frac{\mathrm{d} \alpha}{\mathrm{d} E}\right)_{p=0}
$$

where the first (intrinsic) part corresponds to $\chi^{\mathrm{f}}(\Delta \alpha=0)$ for immobile domain walls and the second (extrinsic) part is the result of domain wall motion. In a similar way, the piezomodulus of a free standing film is

$$
d_{i 3}(\alpha)=\frac{\mathrm{d} \Delta \varepsilon_{i}}{\mathrm{~d} E}=\left(\frac{\partial \Delta \varepsilon_{i}}{\partial p} \frac{\mathrm{d} p}{\mathrm{~d} E}\right)_{\alpha=\alpha_{0}}+\left(\frac{\partial \Delta \varepsilon_{i}}{\partial \alpha} \frac{\mathrm{d} \alpha}{\mathrm{d} E}\right)_{p=0}
$$

$i=3 \quad$ or 1, while $d_{33}^{\mathrm{f}}=d_{33}(\alpha)+\xi d_{31}(\alpha)$ for a constrained film. The susceptibility and piezomodulus of a polydomain film with immobile domain walls are calculated above. The contribution of domain wall movement is considered below.

The changes in the average strain components due to a change in the domain fraction $\Delta \alpha$ are $\Delta \varepsilon_{1}=\frac{1}{2} \Delta \alpha\left(\varepsilon_{c}-\varepsilon_{a}\right), \Delta \varepsilon_{3}=-\Delta \alpha\left(\varepsilon_{c}-\varepsilon_{a}\right)$, which induce bi-axial in-plane stresses as given by

$$
\sigma_{1}=\sigma_{2}=\frac{-\Delta \varepsilon_{1}}{S\left(\alpha_{0}\right)}=\frac{\Delta \alpha\left(\varepsilon_{a}-\varepsilon_{c}\right)}{2 S\left(\alpha_{0}\right)}
$$

Therefore, the free energy corresponding to the change in domain fraction is

$$
\begin{aligned}
\Delta F(\Delta \alpha) & =\frac{\left(\Delta \varepsilon_{1}\right)^{2}}{S\left(\alpha_{0}\right)}-\left(1-\alpha_{0}\right) P_{0} E-\Delta P E \\
& =\frac{\left[\Delta \alpha\left(\varepsilon_{c}-\varepsilon_{a}\right)\right]^{2}}{4 S\left(\alpha_{0}\right)}-\left(1-\alpha_{0}\right) P_{0} E+\Delta \alpha P_{0} E
\end{aligned}
$$

where $\Delta P=-\Delta \alpha P_{0}$ is the change in the saturation polarization of the film due to domain wall movement. The above relation can be obtained from Eq. (6) by noting that $p=0$, and $F^{\mathrm{f}}=0$ for $\alpha=\alpha_{0}$ and $E=0$. The minimization of Eq. (12) with respect to $\Delta \alpha$ yields $\Delta \alpha$ as a function of the applied field, and the change in the saturation polarization due to the field-induced domain wall motion:

$$
\Delta \alpha(E)=-\frac{2 P_{0} S\left(\alpha_{0}\right)}{\left(\varepsilon_{c}-\varepsilon_{a}\right)^{2}} E, \Delta P=\frac{2 P_{0}^{2} S\left(\alpha_{0}\right)}{\left(\varepsilon_{c}-\varepsilon_{a}\right)^{2}} E=\chi^{\mathrm{ext}} E
$$

where $\chi^{\text {ext }}=\frac{8 S\left(\alpha_{0}\right) \chi_{33}^{2}}{\left(d_{33}-d_{31}\right)^{2}}$ is the extrinsic dielectric 
susceptibility of the film due to domain wall movement. The extrinsic piezomodulus in the absence of substrate clamping are given by

$d_{31}^{\mathrm{ext}}=\frac{\partial \varepsilon_{1}}{\partial \Delta \alpha} \frac{\partial \Delta \alpha}{\partial E}=-\frac{P_{0} S\left(\alpha_{0}\right)}{\varepsilon_{c}-\varepsilon_{a}}, d_{33}^{\mathrm{ext}}=\frac{\partial \varepsilon_{3}}{\partial \Delta \alpha} \frac{\partial \Delta \alpha}{\partial E}=\frac{2 P_{0} S\left(\alpha_{0}\right)}{\varepsilon_{c}-\varepsilon_{a}}$

Therefore, the extrinsic contribution to the overall $d_{33}^{\mathrm{f}}$ piezomodulus is

$d_{33}^{\mathrm{f}, \mathrm{ext}}=d_{33}^{\mathrm{ext}}+\xi d_{31}^{\mathrm{ext}}=\frac{P_{0} S\left(\alpha_{0}\right)}{\varepsilon_{c}-\varepsilon_{a}}(2-\xi)=\frac{2 \chi_{33} S\left(\alpha_{0}\right)}{d_{33}(1+\delta)}(2-\xi)$

It is clear that the extrinsic piezomodulus strongly depends on the elastic compliance of the film. The general expressions for the total susceptibility and piezomodulus of the stress-free polydomain film with mobile domain walls are given below in Eqs. (15) and (17), while Eqs. (16) and (18) are the expressions for the specific case of $\alpha_{0}=\alpha_{0}^{*}$ :

$$
\begin{aligned}
& \chi^{\mathrm{f}}\left(\alpha_{0}, \Delta \alpha \neq 0\right) \\
= & \left(1-\alpha_{0}\right) \chi_{33}\left\{\frac{\chi_{33}}{\chi\left(\alpha_{0}\right)}+\frac{2\left[d\left(\alpha_{0}\right)\right]^{2}}{\chi_{33} S\left(\alpha_{0}\right)}\right\}^{-1}+\frac{8 S\left(\alpha_{0}\right) \chi_{33}^{2}}{d_{33}^{2}(1+\delta)^{2}} \\
& \chi^{\mathrm{f}}\left(\alpha_{0}=\alpha_{0}^{*}, \Delta \alpha \neq 0\right)=\frac{1-\delta}{1+\delta} \chi\left(\alpha_{0}^{*}\right)+\frac{8 S\left(\alpha_{0}^{*}\right) \chi_{33}^{2}}{d_{33}^{2}(1+\delta)^{2}} \\
& d_{33}^{\mathrm{f}}\left(\alpha_{0}, \Delta \alpha \neq 0\right) \\
= & \frac{d_{33}\left\{1-\alpha_{0}(1+\delta)+\xi\left[-\delta+\frac{1}{2} \alpha_{0}(1+\delta)\right]\right\}\left(1-\alpha_{0}\right)}{\chi_{33} / \chi\left(\alpha_{0}\right)+2\left[d\left(\alpha_{0}\right)\right]^{2} /\left[\chi_{33} S\left(\alpha_{0}\right)\right]} \\
& +\frac{2 S\left(\alpha_{0}\right) \chi_{33}\left[2-\xi\left(\alpha_{0}\right)\right]}{d_{33}(1+\delta)} \\
d_{33}^{\mathrm{f}}\left(\alpha_{0}=\alpha_{0}^{*}, \Delta \alpha \neq 0\right) & \frac{(1-2 \delta)(1-\delta) \chi\left(\alpha_{0}^{*}\right)}{(1+\delta) \chi_{33}} d_{33}+\frac{2 S\left(\alpha_{0}^{*}\right) \chi_{33}\left[2-\xi\left(\alpha_{0}^{*}\right)\right]}{d_{33}(1+\delta)}
\end{aligned}
$$

\section{Comparison with experimental results}

The experimental results on $1 \mu \mathrm{m}$ thick PZT 20/80 films are presented in Table 1 in comparison with theoretical estimates. The following parameters are used for calculations: $P_{0}=0.7 \mathrm{C} / \mathrm{m}^{2}, Q_{11}=8.14 \times 10^{-2} \mathrm{~m}^{4} / \mathrm{C}^{2}$, $Q_{12}=-2.45 \times 10^{-2} \mathrm{~m}^{4} / \mathrm{C}^{2}, \chi_{11}=1.266 \times 10^{-9}, \chi_{33}=7.65 \times 10^{-10}$, $d_{33}=87.2 \times 10^{-12} \mathrm{C} / \mathrm{N}$ and $d_{31}=-26.2 \times 10^{-12} \mathrm{C} / \mathrm{N}$. It was found that $2\left[d\left(\alpha_{0}\right)\right]^{2} /\left[\chi_{33} S\left(\alpha_{0}\right)\right]<0.1$ for $0.25<\alpha_{0}<$ 0.7. Since the elastic moduli of PZT 20/80 single crystals are unknown, the moduli of $\mathrm{PbTiO}_{3}$ are used for calculations: $S_{11}^{\mathrm{P}}=6.05 \times 10^{-12} \mathrm{~m}^{2} / \mathrm{N}$, $S_{12}^{\mathrm{P}}=-2.65 \times 10^{-12} \mathrm{~m}^{2} / \mathrm{N}, \quad S_{13}^{\mathrm{P}}=-2.30 \times 10^{-12} \mathrm{~m}^{2} / \mathrm{N}$, and $S_{33}^{\mathrm{P}}=10.25 \times 10^{-12} \mathrm{~m}^{2} / \mathrm{N}$ [33]. For films with $\alpha_{0}=0.5$ and $0.6, \quad S\left(\alpha_{0}\right)=4.6 \times 10^{-12} \mathrm{~m}^{2} / \mathrm{N}$ and $4.9 \times 10^{-12} \mathrm{~m}^{2} / \mathrm{N}$, $\tilde{S}\left(\alpha_{0}\right)=-2.48 \times 10^{-12} \mathrm{~m}^{2} / \mathrm{N}$ and $-2.51 \times 10^{-12} \mathrm{~m}^{2} / \mathrm{N}$, and $\xi=1.07$ and 1.03 , respective1y.

It is worthy to note that polydomain islands reveal dramatic increases of polarization and piezoeffect under large electric fields [13,23]. These data as well as direct PFM observations show considerable change of $90^{\circ}$ domain structures, while at weak fields, $\Delta \alpha$, according to Eq. (13), does not exceed 5\%.

\section{Discussion}

The theoretical analysis presented above has not taken into account the contributions of the domain walls and microstresses to the free energy. They are relatively small for films thicker than $300 \mathrm{~nm} \mathrm{[25].} \mathrm{The}$ possibility of the rotation of the polarization in the in-plane domains under an electric field normal to the film has been neglected as well. Therefore, the theoretical analysis cannot pretend to give a quantitative description of experiment results. However, the thermodynamic theory presented here qualitatively explains piezoresponse of continuous constrained films.

For the immobile domain structure, the realistic values of $d_{33}^{\mathrm{f}}$ are bounded by the curves with $\xi=0.8$ and $\xi=2$ in Fig. 4, and therefore, they do not exceed

\begin{tabular}{|c|c|c|c|c|c|c|c|}
\hline \multirow{2}{*}{$\begin{array}{l}\text { Thickness } \\
\text { (nm) }\end{array}$} & \multirow{2}{*}{ Substrate } & \multirow{2}{*}{$\begin{array}{c}a \text {-domain } \\
\text { fraction }(\%)\end{array}$} & \multicolumn{3}{|c|}{$d_{33}$ predicted by theory $(\mathrm{pm} / \mathrm{V})$} & \multicolumn{2}{|c|}{$d_{33}$ in experiment $(\mathrm{pm} / \mathrm{V})$} \\
\hline & & & Intrinsic & Extrinsic & Total & Film & Island \\
\hline 1000 & $\mathrm{SrTiO}_{3}$ & 50 & 20 & 58 & 78 & $60[13]$ & $100-250[13]$ \\
\hline 1000 & $\mathrm{Si}$ & 60 & 14 & 64 & 78 & $50[23]$ & $100-400[23]$ \\
\hline
\end{tabular}
the theoretical values presented in Table 1 by any significant amount. Thus regardless of the elastic moduli of the polydomain film, the intrinsic contribution to its piezoeffect, i.e., the piezoeffect in the absence of domain wall movements, should be considerably less than the experimentally observed

Table 1 Piezomoduli $d_{33}$ of epitaxial polydomain $\mathrm{Pb}\left(\mathrm{Zr}_{0.2} \mathrm{Ti}_{0.8}\right) \mathrm{O}_{3}$ films 
values. Therefore, it is impossible to explain the experiment results shown in Table 1 on continuous constrained films, unless the $90^{\circ}$ domain walls in these films are mobile such that the domain structure changes with the electric field to attain equilibrium.

The theoretical estimations of the extrinsic effect are likely larger than the experimentally observed ones. It can be the result of the homogeneous approximation used for calculations as well as the inaccuracy in the elastic moduli. The extrinsic piezoeffect is proportional to the elastic compliance of the film (see Eq. (14)) and increases with the $a$-domain fraction, while the intrinsic contribution to piezoeffect decreases with the $a$-domain fraction as shown in Fig. 4. Therefore, despite the high mobility of the domain walls, the total piezoeffect of a constrained polydomain film does not exceed much that of a single domain film. On the other hand, there was no discernible change of domain structure observed in a polydomain film under a modest electric field. These two facts help to explain why the extrinsic effect is usually underestimated.

As shown in this paper, the clamping effect on $d_{33}^{\mathrm{f}}$ in polydomain films is more pronounced than in single-domain films. Firstly, because only $c$-domains contribute to the piezoelectric deformation normal to the film/substrate interface in the presence of an electric field parallel to that direction (i.e., the $d_{33}$ piezoelectric mode), the intrinsic piezoeffect of a polydomain film is smaller than that of a single domain film. Secondly, by decreasing polarization in $c$-domains of a polydomain film with fixed domain fraction, the clamping further reduces its intrinsic piezoeffect in comparison with a single domain film under the same electric field. To generally demonstrate the clamping effect on $d_{33}^{\mathrm{f}}$ in polydomain films, an approximation of the piezomodulus given by Eq. (10) is used for the construction of the $d_{33}^{\mathrm{f}}\left(\alpha=\alpha_{0}^{*}, \Delta \alpha=0\right) / d_{33}$ curves in Fig. 4. This is appropriate since the subject of investigation here is the electromechanical response of thick polydomain films, which have domain fractions not too far away from $\alpha_{0}^{*}$.

The second part of the piezoresponse is the extrinsic effect due to the movement of domain walls. It is determined by the elastic interaction between the film and the substrate. The effect of domain wall movement on piezoelectric deformation can be made much more visible if the elastic clamping decreases. One way to decrease clamping is to reduce lateral dimensions of the film to make them comparable to the film thickness, i.e., to microfabricate a ferroelectric thin film island. According to St. Venant principle, the internal stresses due to constraint are located in a region near the island/substrate interface with a depth approximately equal to the lateral dimension of the island. The decrease of the average biaxial stress in such an island can be attributed to the effective increase of its compliance, $S(\alpha)$ (see Eq. (11)). This effective compliance increases with decreasing ratio of the island's lateral size $l$ to the film thickness $h$ and approaches infinity when $l / h$ goes to zero. Therefore, $d_{33}$ of the island approaches $d_{33}$ of a free-standing film or a bulk crystal.

Reducing clamping by decreasing the lateral size of a ferroelectric island leads to the increase of piezoeffect for both single-domain and polydomain films, but the magnitudes of the increase are significantly different. For a single-domain film, $d_{33}$ approaches the value of a bulk crystal as it is observed experimentally $[20,31,34]$. For a polydomain film, the extrinsic effect due to domain wall movement is itself a result of clamping. With decreasing clamping effect, this piezoresponse should increase infinitely. The observed value of $d_{33}$ for the islands $[13,23,35]$ is determined by the remaining clamping effect as well as by finite mobility of domain walls. In this paper we have not considered the effect of elastic domains on nucleation and growth of $180^{\circ}$ domains, which can be important in polydomain ferroelectrics (see, e.g., [36]). Our discussion is focused on the more general phenomena significantly dependent on movement of elastic domains. Therefore, the conclusions obtained here are valid for other polydomain materials. For example, there are experimental evidences of completely relaxed polydomain structures in constrained martensitic films [37,38]. In these films, the change of elastic domain structures compensates the thermal expansion difference between a film and a substrate; thus, the couple is not bent when the temperature is changed.

\section{Conclusions}

Over the past years, it has been widely speculated that the inferior electromechanical and electrical responses of epitaxial polydomain ferroelectric films compared 
to their bulk counterparts are due to the clamping effect of the substrate as well as the immobility of the non- $180^{\circ}$ domain walls. In this article, the theoretical thermodynamic analysis in conjunction with the experiment data in literature clearly demonstrate that elastic domains can be sufficiently mobile in constrained polydomain ferroelectric films, and the relatively small extrinsic piezoeffect in the continuous films can be explained by elastic interactions between the clamped film and substrate. According to thermodynamic predictions, clamping effect can be reduced via micropatterning of the ferroelectric film such that the lateral size of the resulting islands is less than or at least comparable to the film thickness. The reduction of clamping of polydomain films via micropatterning leads to shift of elastic film/substrate equilibrium and dramatic increase of the converse piezoelectric response. Lastly, although this paper is devoted to discussion of ferroelectric films, the general conclusions can be readily applied to constrained polydomain films of shape-memory alloys and ferromagnets.

\section{Acknowledgements}

J. Ouyang would like to acknowledge the "Qi-Lu Young Scholar Fund" (Grant No. 31370080963003) from Shandong University, supported by the 985 Fund of Shandong University, and the project sponsored by SRF for ROCS, SEM (Scientific Research Foundation for the Returned Overseas Chinese Scholars, State Education Ministry). J. Ouyang and W. Zhang would like to thank the financial support of the National Natural Science Foundation of China (Project Grant Nos. 91122024 and 51002088) and the Shandong Province Outstanding Young Scientist Research Fund (Project Grant No. BS2010CL029). A. L. Roytburd is grateful to NSF-DMR (No. 0907122) for the support.

Open Access: This article is distributed under the terms of the Creative Commons Attribution Noncommercial License which permits any noncommercial use, distribution, and reproduction in any medium, provided the original author(s) and source are credited.

\section{References}

[1] Roytburd AL. Martensitic transformation as a typical phase transformation in solids. Solid State Phys 1978,

\section{3: 317-390.}

[2] Roytburd AL. Elastic domains and polydomain phases in solids. Phase Transitions 1993, 45: 1-34.

[3] Khachaturyan AG. Theory of Structural Transformations in Solids. New York: John Wiley \& Sons, 1983.

[4] Roytburd AL, Kosenko NS. Orientational dependence of the elastic energy of a plane interlayer in a system of coherent phases. Phys Status Solidi a 1976, 35: 735-746.

[5] Streiffer SK, Zielinski EM, Lairson BM, et al. Thickness dependence of the twin density in $\mathrm{YBa}_{2} \mathrm{Cu}_{3} \mathrm{O}_{7-\delta}$ thin films sputtered onto $\mathrm{MgO}$ substrates. Appl Phys Lett 1991, 58: 2171-2173.

[6] Ipatova IP, Malyshkin VG, Shchukin VA. Compositional elastic domains in epitaxial layers of phase-separating semiconductor alloys. Philos Mag B 1994, 70: 557-566.

[7] Kaganer VM, Jenichen B, Schippan F, et al. Strain-mediated phase coexistence in heteroepitaxial films. Phys Rev Lett 2000, 85: 341-344.

[8] Kwak BS, Erbil A, Wilkens BJ, et al. Strain relaxation by domain formation in epitaxial ferroelectric thin films. Phys Rev Lett 1992, 68: 3733-3736.

[9] Pompe W, Gong X, Suo Z, et al. Elastic energy release due to domain formation in the strained epitaxy of ferroelectric and ferroelastic films. $J$ Appl Phys 1993, 74: 6012-6019.

[10] Koukhar VG, Pertsev NA, Waser R. Thermodynamic theory of epitaxial ferroelectric thin films with dense domain structures. Phys Rev B 2001, 64: 214103.

[11] Zhang JX, Li YL, Choudhury S, et al. Computer simulation of ferroelectric domain structures in epitaxial $\mathrm{BiFeO}_{3}$ thin films. $J$ Appl Phys 2008, 103: 094111-094111-6.

[12] Ouyang J, Slusker J, Levin I, et al. Engineering of self-assembled domain architectures with ultra-high piezoelectric response in epitaxial ferroelectric films. Adv Funct Mater 2007, 17: 2094-2100.

[13] Nagarajan V, Roytburd A, Stanishevsky A, et al. Dynamics of ferroelastic domains in ferroelectric thin films. Nat Mater 2003, 2: 43-47.

[14] Zhang W, Yuan ML, Wang XY, et al. Design and preparation of stress-free epitaxial $\mathrm{BaTiO}_{3}$ polydomain films by RF magnetron sputtering. Sci Technol Adv Mat 2012, 13: 035006.

[15] Ouyang J, Roytburd AL. Theoretical modeling of coexisting tetragonal and rhombohedral heterophase polydomain structures in lead zirconate titanate ferroelectric films near the morphotropic phase boundary. Acta Mater 2006, 54: 5565-5572. 
[16] Ouyang J, Yang SY, Chen L, et al. Orientation dependence of the converse piezoelectric constants for epitaxial single domain ferroelectric films. Appl Phys Lett 2004, 85: 278-280.

[17] Roytburd AL, Yu Y. In Twinning in Advanced Materials. Yoo MH, Wuttig M, Eds. Warrendale, PA: TMS, 1994, 221: 217.

[18] Pertsev NA, Emelyanov AY. Domain-wall contribution to the piezoelectric response of epitaxial ferroelectric thin films. Appl Phys Lett 1997, 71: 3646-3648.

[19] Alpay SP, Roytburd AL. Thermodynamics of polydomain heterostructures. III. Domain stability map. J Appl Phys 1998, 83: 4714-4723.

[20] Bratkovsky AM, Levanyuk AP. Phase transitions, stability, and dielectric response of the domain structure in ferroelectric-ferroelastic thin films. Phys Rev Lett 2001, 86: 3642-3645.

[21] Bratkovsky AM, Levanyuk AP. Proper ferroelastic phase transitions in thin epitaxial films with symmetry-conserving and symmetry-breaking misfit strains. Phys Rev B 2001, 64: 134107.

[22] Slutsker J, Artemev A, Roytburd AL. Engineering of elastic domain structures in a constrained layer. Acta Mater 2004, 52: 1731-1742.

[23] Ma ZK, Zavaliche F, Chen L, et al. Effect of $90^{\circ}$ domain movement on the piezoelectric response of patterned $\mathrm{PbZr}_{0.2} \mathrm{Ti}_{0.8} \mathrm{O}_{3} / \mathrm{SrTiO}_{3} / \mathrm{Si}$ heterostructures. Appl Phys Lett 2005, 87: 072907.

[24] Roytburd AL, Yu Y. Ferroelectric and ferroelastic domain structures in epitaxial layers. Ferroelectrics 1993, 144: 137-145.

[25] Slutsker J, Artemev A, Roytburd AL. Morphological transitions of elastic domain structures in constrained layers. J Appl Phys 2002, 91: 9049-9058.

[26] Roytburd AL, Alpay SP, Bendersky LA, et al. Three-domain architecture of stress-free epitaxial ferroelectric films. J Appl Phys 2001, 89: 553-556.

[27] Kohli M, Muralt P, Setter N. Removal of $90^{\circ}$ domain pinning in (100) $\mathrm{Pb}\left(\mathrm{Zr}_{0.15} \mathrm{Ti}_{0.85}\right) \mathrm{O}_{3}$ thin films by pulsed operation. Appl Phys Lett 1998, 72:
3217-3219.

[28] Haun MJ, Zhuang ZQ, Furman E, et al. Thermodynamic theory of the lead zirconate-titanate solid solution system, part III: Curie constant and sixth-order polarization interaction dielectric stiffness coefficients. Ferroelectrics 1989, 99: 45-54.

[29] Roytburd AL. Thermodynamics of polydomain heterostructures. I. Effect of Macrostresses. J Appl Phys 1998, 83: 228-238.

[30] Roytburd AL. Thermodynamics of polydomain heterostructures. II . Effect of Microstresses. J Appl Phys 1998, 83: 239-245.

[31] Lefki K, Dormans GJM. Measurement of piezoelectric coefficients of ferroelectric thin films. $J$ Appl Phys 1994, 76: 1764-1767.

[32] Roytburd AL, Alpay SP, Nagarajan V, et al. Measurement of internal stresses via the polarization in epitaxial ferroelectric films. Phys Rev Let 2000, 85: 190-193.

[33] Fesenko EG, Gavrilyachenko VG, Semenchev AF. Domain Structure of Multiaxial Ferroelectric Crystals. Rostov-on-Don: Rostov University Press, 1990.

[34] Nagarajan V, Stanishevsky A, Chen L, et al. Realizing intrinsic piezoresponse in epitaxial submicron lead zirconate titanate capacitors on $\mathrm{Si}$. Appl Phys Lett 2002, 81: 4215-4217.

[35] Bühlmann S, Dwir B, Baborowski J, et al. Size effect in mesoscopic epitaxial ferroelectric structures: Increase of piezoelectric response with decreasing feature size. Appl Phys Lett 2002, 80: 3195-3197.

[36] Ganpule CS, Roytburd AL, Nagarajan V, et al. Polarization relaxation kinetics and $180^{\circ}$ domain wall dynamics in ferroelectric thin films. Phys Rev B 2001, 65: 014101.

[37] Roytburd AL, Kim TS, Su QM, et al. Martensitic transformation in constrained films. Acta Mater 1998, 46: 5095-5107.

[38] Mathews SA. Ph.D.Thesis. University of Maryland, 2000 . 